\title{
2,5-二取代-1,3,4-噁二唑不对称弯曲形液晶合成与性能研究
}

\author{
毛志鹏 ${ }^{a}$ 游红军 ${ }^{a}$ 王 坤 ${ }^{a}$ 张智勇*, $a$ \\ 关金涛 ${ }^{a}$ 戴志群 $a$ 项 颖 ${ }^{b}$ \\ ( ${ }^{a}$ 武汉轻工大学化学与环境工程学院 武汉 430023)

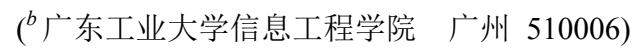

\begin{abstract}
摘要 以 2,5-二取代-1,3,4-噁二唑为中心核, 酯基 $(\mathrm{COO})$ 为桥键, 烷基 $(n=5 \sim 9)$ 为末端基团, 在单侧苯环侧位上引入氟 原子, 设计合成了两个系列新型不对称弯曲形液晶化合物 9a $\sim 9 \mathbf{e}$ 和 10a $\sim 10$; 并通过 $I R,{ }^{1} \mathrm{H} \mathrm{NMR},{ }^{13} \mathrm{C} N \mathrm{~N}$, ${ }^{19} \mathrm{~F} N \mathrm{~N} R$ 和 MS 对其结构进行鉴定, 经差热扫描仪(DSC)和偏光显微镜(POM)测定其液晶相态. 结果表明: 所有目标化合物的分 子结构正确, 都呈现向列相态, 向列相态温度范围最宽达 $78{ }^{\circ} \mathrm{C}$; 侧位单氟苯环结构化合物 $9 \mathrm{a} \sim 9 \mathrm{e}$ 的向列相态温度范 围没有二氟苯环结构 10a 10e 的宽, 并且化合物 9a $\sim 9 \mathrm{e}$ 都呈现奇偶效应.
\end{abstract}

关键词＼cjkstart噁二唑; 不对称弯曲形液晶; 向列相; 合成

\section{Synthesis and Property of Unsymmetric Bent-core Liquid Crystals Based on 2,5-Disubstitueted-1,3,4-oxadiazole}

\author{
Mao, Zhipeng ${ }^{a} \quad$ You, Hongjun $^{a} \quad$ Wang, Kun $^{a} \quad$ Zhang, Zhiyong $^{* a}$
}

Guan, Jintao $^{a} \quad$ Dai, Zhiqun $^{a} \quad$ Xiang, Ying $^{b}$

( ${ }^{a}$ Department of Chemistry and Environmental Engineering, Wuhan Polytechnic University, Wuhan 430023)

( ${ }^{b}$ Department of Technology Institute and Information Engineering, Guangdong Polytechnic University, Guangzhou 510006)

\begin{abstract}
Two novel series of bent-core liquid crystals $(9 \mathbf{a} \sim 9 \mathrm{e}$ and $\mathbf{1 0 a} \sim 10 \mathrm{e})$ were prepared with a central bent core based on 2,5-disubstitueted-1,3,4-oxadiazole, ester group bridge (coo), alkyl tails with different carbon numbers $(n=5 \sim 9)$, and one side wing substituted by fluorine atom. The structures of the target compounds were confirmed by IR, ${ }^{1} \mathrm{H}$ NMR, ${ }^{13} \mathrm{C} \mathrm{NMR}$, $\mathrm{MS}$, and their transition temperatures and phase textures were investigated by using differential scanning calorimetric (DSC) and polarizing optical microscopy (POM). The result suggested that all target compounds had right chemical structure. All compounds 9a $\sim 9 \mathrm{e}$ and 10a $\sim 10 \mathrm{e}$ exhibited nematic phase and existed wide temperature range of nematic phase. The widest range of nematic phase was $78{ }^{\circ} \mathrm{C}$. The temperature range of nematic phase of compounds $9 \mathbf{9 a} \sim 9 \mathrm{e}$ with lateral monofluoro-substituted benzene was more narrow than compounds $10 \mathrm{a} \sim 10 \mathrm{e}$ with lateral difluoro-substituted benzene. Moreover, compounds 9a $\sim 9 e$ showed odd-even effect.
\end{abstract}

Keywords oxadiazole; unsymmetric bent-core liquid crystal; nematic phase; synthesis

弯曲形(或叫香蕉型)液晶是近年来发现的一类具有 独特光电性能的新型液晶材料, 它除了一条光学主轴 外, 还存在两条与主轴垂直的光学副轴, 而且在两个光 学副轴方向上也呈现明显的光学各向异性. 1996 年 Nori 等 ${ }^{[1]}$ 首次报道了一类非手性的香蕉型分子具有铁电性 (后经证实是反铁电性); 1997 年 Link 等 ${ }^{[2]}$ 也证实了这一 结果, 并且认为这类非手性分子的旋光性是由于分子倾
斜层状堆积造成, 并发现用电场来调控的铁电性与反铁 电性是互变的, 也因此掀起了一股弯曲形液晶的研究热 潮. 2004 年 Madsen 等 ${ }^{[3]}$ 合成出第一个真正意义上的热致 双轴向列相液晶化合物 $\mathbf{1}$, 其向列相范围达到 $49{ }^{\circ} \mathrm{C}$, 但 熔点和向列相转变温度太高, 且近晶相相态较多(图 1).

2005 年 Prasad 等 ${ }^{[5]}$ 报道了一类以 1,3 -二取代苯为中 心环的席夫碱弯曲形液晶分子, 出现了具有显光电示价

\footnotetext{
* E-mail: zzy6211@126.com

Received April 18, 2015; revised July 5, 2015; published online August 25, 2015.

Project supported by the National Natural Science Foundation of China (Nos. 11074054, 11374067).

国家自然科学基金(Nos. 11074054, 11374067)资助项目.
} 


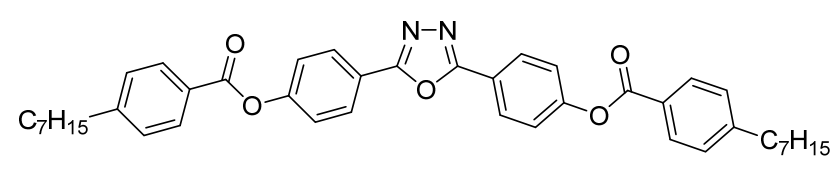

(Cr $148{ }^{\circ} \mathrm{C}$ SmY $166{ }^{\circ} \mathrm{C} \operatorname{SmX} 173{ }^{\circ} \mathrm{C} N 222{ }^{\circ} \mathrm{C}$ Iso)

图 1 弯曲形液晶化合物 1 结构式

Figure 1 The structure of compound 1

值的向列相液晶态, 但因相变温度太高而无法进行应用 研究. 近年来人们通过改变中心基团、桥键、末端链或 引入侧位取代以求获得低熔点弯曲型液晶 ${ }^{[5,6,7]} ; 2009$ 年 Görtz 等 ${ }^{[8]}$ 以噁二唑为中心合成了两个具有侧氟取代的 苯甲酸酯类弯曲形液晶, 降低了液晶的熔点和向列相转 变温度; 同年 Cooper 等 ${ }^{[9]}$ 合成了一系列对称的侧位甲基 取代啞二唑苯甲酸酯类弯曲形液晶, 其中侧位双甲基取 代的液晶化合物的相列相转变温度得到了大幅度降低, 最大降幅达 $87{ }^{\circ} \mathrm{C} .2012$ 年 Speetjens 等 ${ }^{[10]}$ 报道合成了一 系列不对称侧位三甲基取代的噁二唑苯甲酸酯类弯曲 液晶化合物, 其向列相转变温度降低到 $100{ }^{\circ} \mathrm{C}$ 以下; 2013 年王勇丽、杜琼等 ${ }^{[11]}$ 以噁二唑为弯曲中心, 以亚甲 氧基 $\left(\mathrm{CH}_{2} \mathrm{O}\right)$ 为桥键合成了一系列新型弯曲液晶, 这类 弯曲液晶都具有较低熔点, 大部分在 $100{ }^{\circ} \mathrm{C}$ 以下, 最低 达 $80.6{ }^{\circ} \mathrm{C}$, 但这些化合物没有呈现向列相, 而具有向 列相态的弯曲液晶是应用发展研究的基础. 本文以 2,5二取代-1,3,4-惡二唑为中心核, 酯键为桥键, 直链烷基 为末端基团, 单臂苯环侧位引入氟原子, 设计合成了两
个系列非对称弯曲形液晶化合物 $9 \mathrm{a} \sim 9 \mathrm{e}$ 和 $10 \mathrm{a} \sim 10 \mathrm{e}$, 以期获得宽温向列相态、降低熔点的弯曲液晶材料, 其 中化合物 $9 \mathrm{c}$ 和 $10 \mathrm{c}$ 已有文献报道 ${ }^{[8]}$. 目标化合物合成路 线见 Scheme 1.

\section{1 结果与讨论}

本文合成了 10 个新型目标化合物 9a $\sim 9 \mathrm{e}$ 和 $10 \mathrm{a} \sim$ 10e, 经过 IR、 ${ }^{1} \mathrm{H} N M R 、{ }^{13} \mathrm{C}$ NMR、 ${ }^{19} \mathrm{~F}$ NMR 和 MS 对 其进行表征, 结果表明这些化合物的分子结构正确. 目 标化合物相态的确定，首先通过 TA 公司 Q-10 型差热分 析扫描仪测定目标化合物各个相态的温度区间, 升温速 率为 $5{ }^{\circ} \mathrm{C} / \mathrm{min}$; 再通过 XPN-100 E 投射偏光熔点仪观察 每个化合物在各个温度区间内的相态结构, 由此确定每 个化合物在不同温度区间内的相态种类.

\section{1 目标化合物 $9 a \sim 9 e$ 和 $10 a \sim 10 e$ 的液晶相态分析}

表 1 是目标化合物 $9 \mathrm{a} \sim 9 \mathrm{e}$ 的相变温度和焓变数据. 由表 1 可以看出：化合物 $9 \mathrm{a} \sim 9 \mathrm{e}$ 都具有近晶相态和向列 相态, 近晶相态温度范围较宽, 其中化合物 $9 d$ 的近晶相 变温度范围竟有 $73{ }^{\circ} \mathrm{C}$; 而向列相的温度范围相对较窄, 最宽为 $56{ }^{\circ} \mathrm{C}(9 \mathrm{c})$, 最窄为只有 $28{ }^{\circ} \mathrm{C}(9 \mathrm{e})$. 并且随着 末端烷基链的碳数增多, 这些化合物的向列相温度范围 也逐渐变窄, 近晶相态却不断增加, 不仅出现多种近晶 相态，而且近晶相态温度范围比向列相态宽一倍，如化 合物 9d 和 9e(图 2). 这些化合物的相变温度、熔点以及

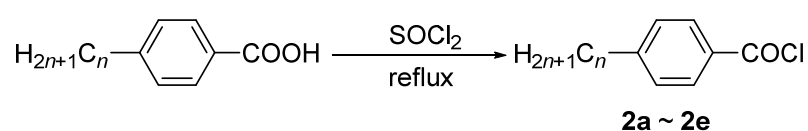

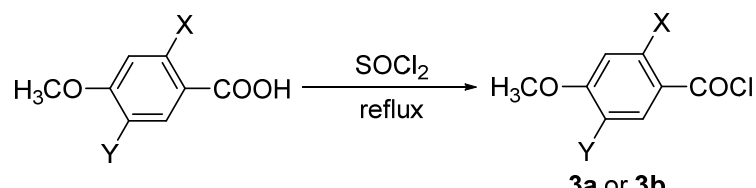
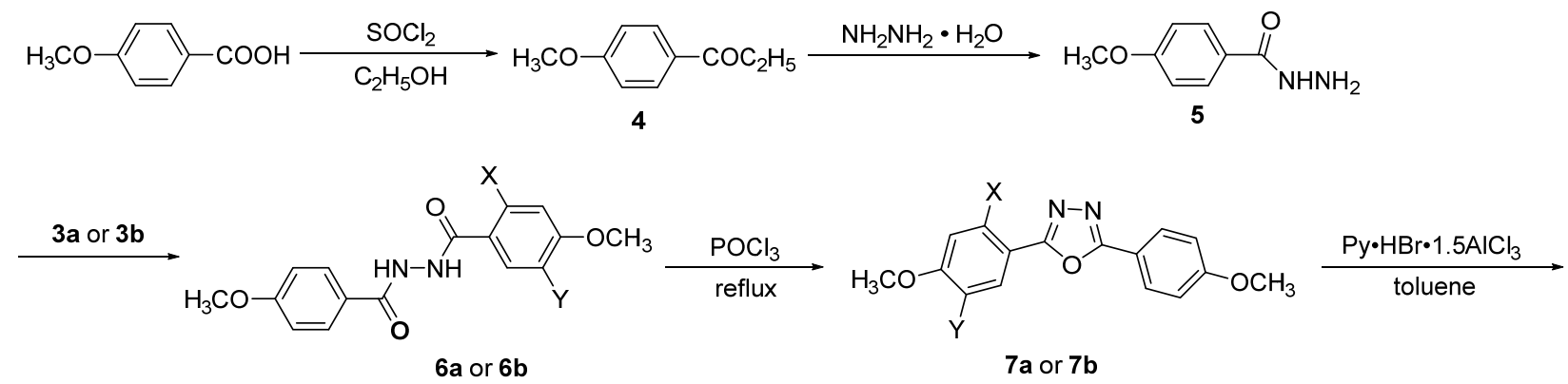<smiles>[Y]c1cc(-c2nnc(-c3ccc(O)cc3)o2)c([X])cc1O</smiles><smiles>C[10BH]C</smiles>

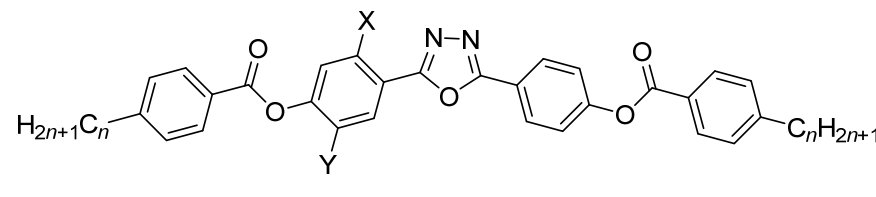

$9 a \sim 9 e$ or $10 a \sim 10 e$

9: $\mathrm{X}=\mathrm{F}, \mathrm{Y}=\mathrm{H} ; \quad$ 10: $\mathrm{X}=\mathrm{F}, \mathrm{Y}=\mathrm{F} ; n=5,6,7,8,9$

图式 1 目标化合物的合成路线

Scheme 1 The synthetic routes of target compounds 
表 1 目标化合物 $9 \mathrm{a} \sim 9 \mathrm{e}$ 的相变温度和焓变 ${ }^{a}$

Table 1 Phase transition temperatures and enthalpy changes of target compounds $9 \mathbf{a} \sim 9 \mathrm{e}$

\begin{tabular}{|c|c|c|c|}
\hline Compd. & $n$ & Phase transition temperatures $\left({ }^{\circ} \mathrm{C}\right)$ and enthalpy $(\mathrm{J} / \mathrm{g})$ & $\Delta T_{\mathrm{N}} /{ }^{\circ} \mathrm{C}$ \\
\hline 9a & 5 & Cr 150 (62.19) Sm 192 (1.35) N 239 (1.10) Iso & 47 \\
\hline 9b & 6 & Cr 113 (37.06) Sm 137 (58.21) N 175 (1.10) Iso & 38 \\
\hline $9 c$ & 7 & Cr 137 (34.40) SmI 151 (7.50) SmII $162(21.80)$ N 218 (2.35) Iso & 56 \\
\hline 9d & 8 & Cr 94 (18.26) SmI 129 (9.76) SmII 144 (6.15) SmIII 169 (17.84) N 202 (2.20) Iso & 33 \\
\hline $9 e$ & 9 & Cr 127 (21.82) SmI 142 (3.92) SmII 167 (12.86) N 195 (0.65) Iso & 28 \\
\hline
\end{tabular}

${ }^{a} \mathrm{Cr}$ 为晶体相, $\mathrm{Sm}$ 为近晶相, $\mathrm{N}$ 为向列相, Iso 为各向同性液体, $\Delta T_{\mathrm{N}}$ 为向列相温度范围.

清亮点都随着烷基碳数变化而呈现奇偶效应现象, 奇数 碳链分子的熔点和清亮点往往高于偶数碳链分子.

表 2 是目标化合物 $10 \mathrm{a} \sim 10 \mathrm{e}$ 的相变温度和焓变数 据. 由表 2 可以看出: 系列化合物 $10 \mathrm{a} \sim 10 \mathrm{e}$ 都呈现向列 相, 且相列相温度范围较宽, 最宽达 $78{ }^{\circ} \mathrm{C}(\mathbf{1 0 b})$, 随着 末端基团碳链的增长, 向列相温度范围变窄; 该系列化 合物近晶相态较少, 只有 $10 \mathrm{a}$ 和 $10 \mathrm{c}$ 存在近晶相, 且近 晶相温度范围较窄(图 2).

表 2 目标化合物 $10 \mathrm{a} \sim 10 \mathrm{e}$ 的相变温度和焓变 ${ }^{a}$

Table 2 Phase transition temperatures and enthalpy changes of target compounds $\mathbf{1 0 a} \sim \mathbf{1 0 e}$

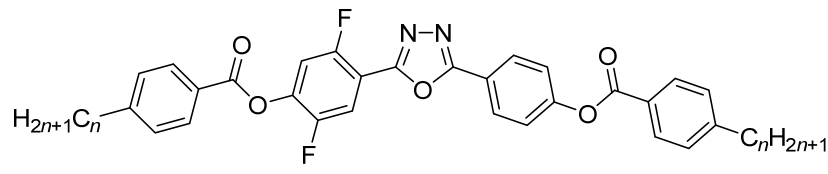

\begin{tabular}{|c|c|c|c|}
\hline Compd. & $n$ & $\begin{array}{l}\text { Phase transition temperatures }\left({ }^{\circ} \mathrm{C}\right) \\
\text { and enthalpy }(\mathrm{J} / \mathrm{g})\end{array}$ & $\Delta T_{\mathrm{N}} /{ }^{\circ} \mathrm{C}$ \\
\hline $10 a$ & 5 & $\begin{array}{l}\text { Cr } 158(70.01) \text { Sm } 199(1.10) \mathrm{N} \\
239 \text { (1.34) Iso }\end{array}$ & 40 \\
\hline 10b & 6 & Cr $142(54.13) \mathrm{N} 220$ (1.24) Iso & 78 \\
\hline $10 \mathrm{c}$ & 7 & $\begin{array}{l}\text { Cr } 121(10.51) \mathrm{Sm} 141(48.16) \mathrm{N} \\
213(0.80) \text { Iso }\end{array}$ & 72 \\
\hline 10d & 8 & Cr 139 (58.37) N 196 (0.68) Iso & 57 \\
\hline 10e & 9 & Cr $144(46.54) \mathrm{N} 198(0.88)$ Iso & 54 \\
\hline
\end{tabular}

单氟取代化合物 9a 9e 的近晶相的温度范围明显 比二氟取代化合物 10a 10e 的宽, 且近晶相种类多; 系 列 9 的向列相范围(除 5 个碳)明显比系列 10 要窄. 但它 们的熔点都随着末端烷基链的增长而降低, 这主要是因 为烷基链增长, 分子柔性增加, 造成分子间紧密堆积, 从而减小分子之间相互作用力, 使得熔点降低; 与此同 时, 随着末端链的增长, 同时也增加了末端烷基链与中 心极化部分之间的距离, 使得液晶分子的局部极化率减 小, 从而导致液晶分子的侧向引力大于末端引力, 易出 现近晶相; 不仅如此, 向列相范围随着末端碳链增长逐
渐缩小, 但系列化合物 10 的向列相范围没有系列化合 物 9 变化得那么明显(图 2). 这可能是由于 $10 \mathrm{a} \sim 10 \mathrm{e}$ 比 9a 9e 分子的刚性臂上碳-氟键作用的结果.

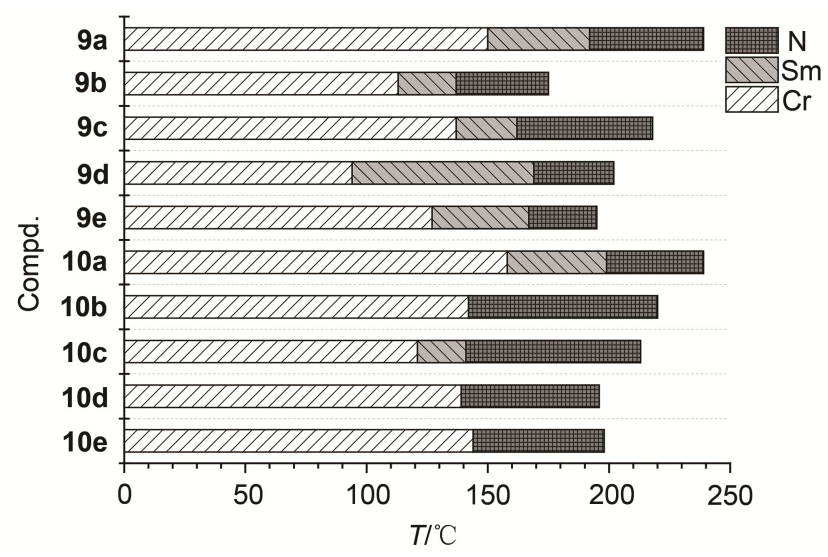

图 2 化合物 9a $\sim 9 \mathrm{e}$ 和 $10 \mathrm{a} \sim 10 \mathrm{e}$ 的相变温度分布情况

Figure 2 The phase distribution of compounds $9 \mathbf{a} \sim 9 \mathrm{e}$ and $10 \mathrm{a} \sim 10 \mathrm{e}$

\section{2 侧氟取代对液晶熔点和相态的影响}

任何液晶分子的性能都与其结构都有着紧密的联 系, 比较烷基碳数相同、化学结构相近的化合物 $\mathbf{1}^{[3]} 、 9 \mathrm{c}$ 和 $10 \mathrm{c}$ (表 3); 化合物 1 是没有被氟取代的分子，化合物 9c 为单氟取代, 化合物 10c 为二氟取代, 且两个氟原子 处于侧臂苯环对位, 使其中心环部分成为非对称的氟取 代苯环体系. 实验结果表明: 化合物 $9 \mathrm{c}$ 和 $10 \mathrm{c}$ 的熔点和 向列相转变温度降低, 向列相温度范围随着氟原子增加 而不断加宽. 与化合物 1 相比, 化合物 $9 \mathrm{c}$ 和 $10 \mathrm{c}$ 的熔点 分别降低了 11 和 $27{ }^{\circ} \mathrm{C}$ 左右, 向列相转变温度也分别降 低了 11 和 $21{ }^{\circ} \mathrm{C}$, 向列相的温度范围由 $49{ }^{\circ} \mathrm{C}$ 分别拓宽 到了 56 和 $71{ }^{\circ} \mathrm{C}$, 分别增加了 7 和 $22{ }^{\circ} \mathrm{C}$. 可见, 氟原子 的引入, 不仅有利于降低化合物的熔点和向列相转变温 度, 也有利于拓宽了液晶化合物的向列相温度范围, 而 且随着弯曲分子刚性臂上侧位氟原子数目的增加, 碳氟键(氟原子)使分子间的侧向引力和相互作用力不断减 
小，近晶相不断压缩，向列相范围不断拓宽.

表 3 化合物 $1 、 9 \mathrm{c}$ 和 $10 \mathrm{c}$ 的相变温度比较 ${ }^{a}$

Table 3 Phase transition temperatures of compounds 1, 9c and 10c

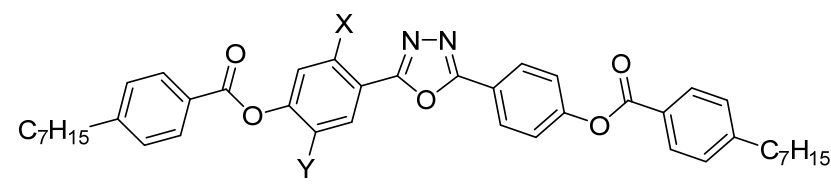

\begin{tabular}{ccclc}
\hline Compd. & $\mathrm{X}$ & $\mathrm{Y}$ & $\begin{array}{c}\text { Phase transition } \\
\text { temperature } /{ }^{\circ} \mathrm{C}\end{array}$ & $\Delta T_{\mathrm{N}} /{ }^{\circ} \mathrm{C}$ \\
\hline $\mathbf{1}$ & $\mathrm{H}$ & $\mathrm{H}$ & $\begin{array}{l}\mathrm{Cr} 148 \mathrm{SmI} 166 \mathrm{SmII} \\
173 \mathrm{~N} 222 \mathrm{Iso}\end{array}$ & 49 \\
9c & $\mathrm{F}$ & $\mathrm{H}$ & $\begin{array}{l}\mathrm{Cr} 137 \mathrm{SmI} 151 \mathrm{SmII} \\
162 \mathrm{~N} 218 \mathrm{Iso}\end{array}$ & 56 \\
10c & $\mathrm{F}$ & $\mathrm{F}$ & $\mathrm{Cr} 121 \mathrm{Sm} \mathrm{141} \mathrm{N} \mathrm{213} \mathrm{Iso}$ & 72 \\
\hline
\end{tabular}

${ }^{a} \mathrm{Cr}$ 为晶体相, $\mathrm{Sm}$ 为近晶相, $\mathrm{N}$ 为向列相, Iso 为各向同性液体, $\Delta T_{\mathrm{N}}$ 为向列 相温度范围

\section{3 化合物 9c 和 10a 的相态结构图分析}

图 3 为目标化合物 10a 的 DSC 曲线和 POM 结构图. 由图 3a 可知, 化合物 10a 在 $158{ }^{\circ} \mathrm{C}$ 进入液晶态, 由晶体 相转变为近晶相, 即熔点为 $158{ }^{\circ} \mathrm{C}$; 加热到 $198{ }^{\circ} \mathrm{C}$ 时, 由近晶相转变为向列相, 一般而言, 近晶相转化为向列 相的热涵变化较大, 但弯曲液晶化合物 $\mathbf{1 0 a}$ 在 $198{ }^{\circ} \mathrm{C}$ 时 的热涵变很小, 这个问题有待进一步研究确定; 加热到 $239{ }^{\circ} \mathrm{C}$ 时, 由向列相转变为各向同性态, 即清亮点. 图 $3 \mathrm{~b}$ 为目标化合物 10a 升温过程中在 $178{ }^{\circ} \mathrm{C}$ 时拍到的偏 光显微照片, 属于近晶相的结构; 图 $3 \mathrm{c}$ 为目标化合物 10a 升温过程中在 $230{ }^{\circ} \mathrm{C}$ 时的偏光显微照片, 属于向列 相的结构.

图 4 是化合物 $9 \mathrm{c}$ 在升温过程中, 不同温度下的相态 结构变化情况. 化合物 9c 在 $141 、 145$ 和 $155{ }^{\circ} \mathrm{C}$ 时分别 是三个不同的近晶相态结构 SmI(图 4a)、SmII(图 4b), $\operatorname{SmIII}\left(\right.$ 图 4c); 而在 $162{ }^{\circ} \mathrm{C}$ 以上随着温度增加, 出现一系 列的单色区域色彩变化的丝状结构, 这种相态结构是向 列相态, 并具有手性向列相态的特征结构特点, 这种相 态结构与近年为众多液晶工作所亲睐的蓝相液晶极为 相似, 也有可能是蓝相液晶的相态结构(见图 $4 \mathrm{~d} 、 4 \mathrm{e}$ 所 示), 需要通过其它手段对液晶相态进一步鉴定研究; 图 4f 是典型的向列相态结构.

\section{2 结论}

本文设计合成了两个系列共 10 个噁二唑不对称弯 曲形液晶化合物 $9 \mathrm{a} \sim 9 \mathrm{e}$ 和 10a $\sim 10 \mathrm{e}$, 所有目标化合物 都经差热扫描仪(DSC)和偏光显微镜(POM)测定其液晶 相态. 结果表明: 这些化合物都能呈现液晶相态, 而且 呈现出较宽温度范围的向列相, 最宽达 $78{ }^{\circ} \mathrm{C}(\mathbf{1 0 c})$; 侧
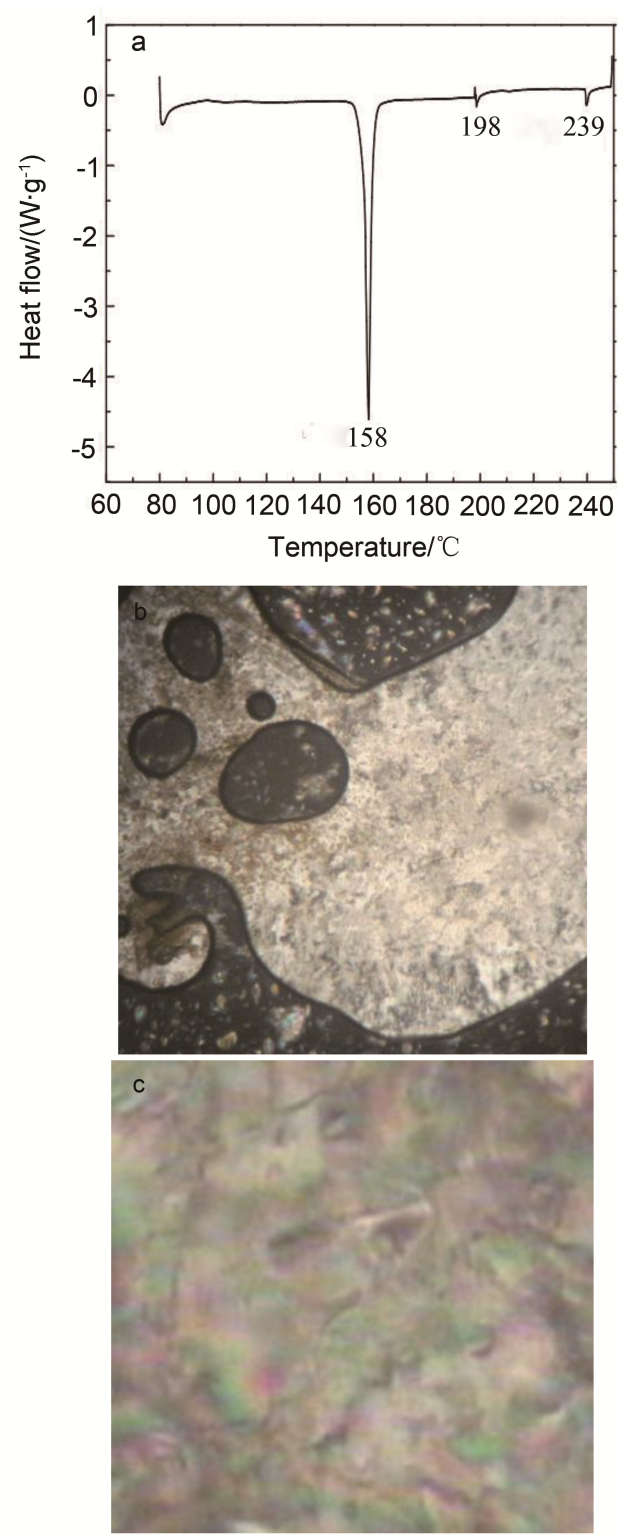

图 3 目标化合物 10a 的 DSC 曲线(a)及在 $178{ }^{\circ} \mathrm{C}$ 时的近晶相 态结构 $(b)(100 \times)$ 和在 $230{ }^{\circ} \mathrm{C}$ 时的向列相相态结构 $(\mathrm{c})(100 \times)$ Figure 3 DSC curve of target compound 10a (a), the smectic textures at $178{ }^{\circ} \mathrm{C}(100 \times)$ (b) and the nematic textures at $230{ }^{\circ} \mathrm{C}(100 \times)(\mathrm{c})$

位氟原子的引入，可以降低液晶的熔点，与没有侧氟取 代且末端基团都为正庚基的液晶化合物 1 做比较, 9c 和 $10 \mathrm{c}$ 的熔点分别降低了 11 和 $27{ }^{\circ} \mathrm{C}$; 化合物 10a $~ 10 \mathrm{e}$ 比 9a 9e 的相列相范围宽，这是因为随着侧位氟原子取代 数目的增加, 可以减小分子间的侧向引力，压缩近晶相, 拓宽向列相范围; 随着液晶分子末端烷基链的增长，分 子之间容易滑动产生近晶相，压缩向列相范围; 系列化 合物 9a 9e 表现出明显的奇偶效应, 奇数碳链液晶分 子的熔点大于偶数碳链. 为今后发展弯曲液晶化合物及 其双轴液晶材料提供了素材和思路。 

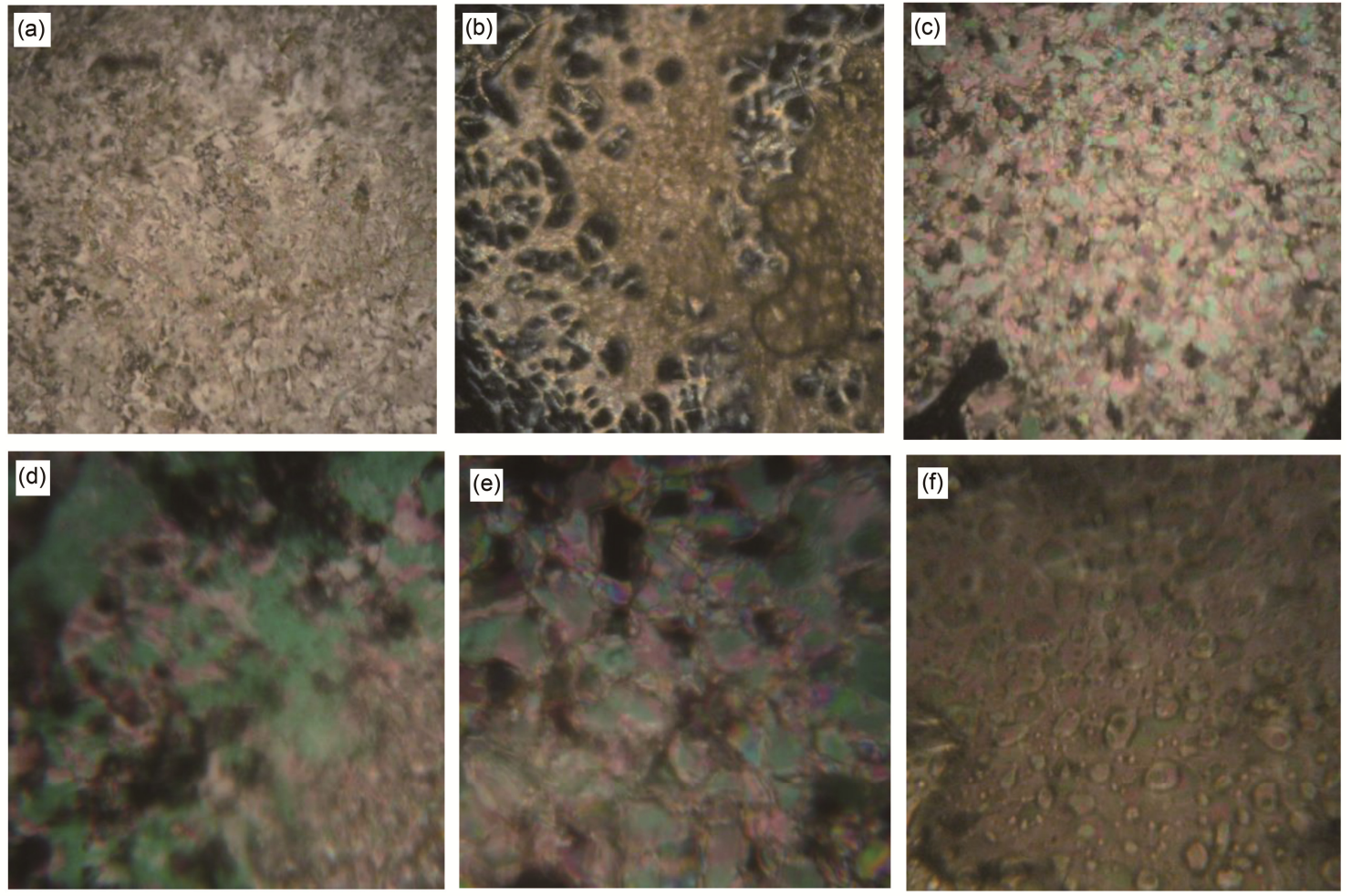

图 4 化合物 $9 \mathrm{c}$ 在不同温度下观察到的液晶相态光学结构 $(\times 100)$

Figure 4 Optical textures of compound $9 c$ under different temperature $(\times 100)$

\section{3 实验部分}

\section{1 仪器与试剂}

Thermo 公司 330 FT-IR 型红外光谱仪; Thermo 公 司 LTQ XL 型质谱仪, ESI 离子源 $(35 \mathrm{eV})$, 气源为高纯度 氮气(纯度>99.999\%); Varian 公司 INOVO $400 \mathrm{MHz}$ 核 磁共振仪(TMS 内标); 北京泰克公司 X-4 型数字显微熔 点仪; TA 公司 Q-10 型差热分析扫描仪.

4-正戌基苯甲酸(99.5\%), 河北美星化工有限公司; 4-甲氧基苯甲酸(CP), 国药集团化学试剂有限公司; 2 氟-4-甲氧基苯甲酸(99.5\%), 石家庄市康博精细化工有 限公司; 其他试剂均为化学纯, 试剂公司购买; 2,5 -二 氟-4-甲氧基苯甲酸、4-正己基苯甲酸、4-正庚基苯甲酸、 4-正辛基苯甲酸和 4-正壬基苯甲酸均是本实验室合成.

\section{2 实验方法}

\section{2 .14 -正烷基苯甲酰氯 $(2 \mathbf{a} \sim 2 \mathbf{e})$ 的合成}

在装有回流冷凝管和干燥管的 $250 \mathrm{~mL}$ 的单口圆底 烧瓶中加入 4-正烷基苯甲酸(0.1 mol)和 $\mathrm{SOCl}_{2}(50 \mathrm{~mL})$, 回流 $4 \mathrm{~h}$. 常压蒸出 $\mathrm{SOCl}_{2}$, 再加入甲苯 $(10 \mathrm{~mL} \times 2)$ 减压 蒸出残余的 $\mathrm{SOCl}_{2}$, 得棕色液体粗品 $\mathbf{2 a} \sim 2 \mathbf{e}^{[12]}$, 备用.

同理合成 2-氟-4-甲氧基苯甲酰氯(3a) 和 2,5-二氟-4甲氧基苯甲酰氯(3b).

\subsubsection{4-甲氧基苯甲酸乙酯(4)的合成}

在装有机械搅拌, $250 \mathrm{~mL}$ 恒压滴液漏斗、带干燥管 的回流冷凝管和温度计的 $500 \mathrm{~mL}$ 三口瓶中加入对甲氧 基苯甲酸 $(91.3 \mathrm{~g}, 0.6 \mathrm{~mol})$ 和无水乙醇 $(300 \mathrm{~mL})$, 冰盐浴 降温至 $-5{ }^{\circ} \mathrm{C}$. 缓慢滴加 $\mathrm{SOCl}_{2}(100 \mathrm{~mL})$, 控制温度不 超过 $0{ }^{\circ} \mathrm{C}$; 滴毕, 自然升至室温, 再加热回流 $8 \mathrm{~h}$. 停止 加热, 冷至室温; 将反应混合液倒入冰水 $(200 \mathrm{~mL})$ 中充 分搅拌; 萃取分液, 用乙酸乙酯 $(100 \mathrm{~mL} \times 3)$ 提取有机 层; 合并有机层, 用 $10 \%$ 的 $\mathrm{Na}_{2} \mathrm{CO}_{3}$ 溶液洗至中性. 蒸干 溶剂, 油泵减压蒸馏, 得 $95.5 \mathrm{~g}$ 无色透明液体 $\mathbf{4}^{[13]}$, 产率 $92.2 \%$. b.p. $128 \sim 130{ }^{\circ} \mathrm{C} / 667 \mathrm{~Pa}$ (文献值 ${ }^{[13]}: 263{ }^{\circ} \mathrm{C}$ ).

\subsubsection{4-甲氧基苯甲酰肼(5)的合成}

在装有回流冷凝管的 $500 \mathrm{~mL}$ 三口圆底烧瓶中加入 化合物 4 (97.3 g, $0.54 \mathrm{~mol})$ 、水合肼(40.6 g, $0.65 \mathrm{~mol})$ 和 无水乙醇 $(140 \mathrm{~mL})$, 升温至 $80{ }^{\circ} \mathrm{C}$ 回流 $12 \mathrm{~h}$. 停止加热, 稍冷后, 蒸干溶剂, 抽滤, 水洗至中性. 烘干, 得 $70.1 \mathrm{~g}$ 白色针状晶体产物 $5^{[14,15]}$, 产率 78.1\%. m.p. 104 $105{ }^{\circ} \mathrm{C}$ (文献值 ${ }^{[14]}$ : $104 \sim 106{ }^{\circ} \mathrm{C}$ ); IR (KBr) v: 3332, 3056, 2937, 2839, 1619, 1575, 1496, 1443, 1341, 1326, $1257,1186,1174,1037,930,845,609 \mathrm{~cm}^{-1}$.

2.2 .42 -氟-4-甲氧基- $N$ '-(4-甲氧基苯甲酰基)苯甲酰肼 (6a) 的合成

在装有 $100 \mathrm{~mL}$ 恒压滴液漏斗、回流冷凝管和温度 
计的 $100 \mathrm{~mL}$ 三口圆底烧瓶中加入化合物 $5(8.3 \mathrm{~g}, 0.05$ $\mathrm{mol}$ )和 $70 \mathrm{~mL}$ 干燥过的吡啶, 搅拌, 冰盐浴冷却至 $5{ }^{\circ} \mathrm{C}$, 缓慢滴加化合物 3a ( $9.4 \mathrm{~g}, 0.05 \mathrm{~mol})$ 的甲苯 $(30 \mathrm{~mL})$ 溶液, 控制温度在 $10{ }^{\circ} \mathrm{C}$ 以下. 反应 $2 \mathrm{~h}$ 后, 自然升至室温, 再 缓慢加热到 $90{ }^{\circ} \mathrm{C}$, 反应至瓶内固体全部溶解. 停止加 热, 冷却至室温, 水泵减压蒸出大部分吡啶, 残余液倒 入冰水中搅拌, 静置. 抽滤, 烘干, 无水乙醇重结晶, 得 到 $12.5 \mathrm{~g}$ 白色针状晶体 $\mathbf{6 a}^{[14]}$, 产率 78.6\%. m.p. 215 $216{ }^{\circ} \mathrm{C}$; IR (KBr) v: 3226, 1635, 1605, 1585, 1512, 1476, $1355,1288,1180,1027,1012,841,752,607 \mathrm{~cm}^{-1}$.

同理合成 2,5-二氟-4-甲氧基- $N^{\prime}$-(4-甲氧基苯甲酰 基)苯甲酰肼(6b), 得 $12.7 \mathrm{~g}$ 白色针状晶体, 产率 75.3\%. m.p. $234 \sim 236{ }^{\circ} \mathrm{C}$; IR (KBr) v: 3224, 1632, 1603, 1582, 1515, 1471, 1350, 1288, 1254, 1179, 1027, 1012, 841, 800, $752,605 \mathrm{~cm}^{-1}$.

2.2.5 2-(2-氟-4-甲氧基苯基)-5-(4-甲氧基苯基)-1,3,4噁二唑 $(7 a)$ 的合成

在装有回流冷凝管、干燥管和温度计的 $250 \mathrm{~mL}$ 三 口圆底烧瓶中, 加入化合物 6a (12.5 g, $0.0393 \mathrm{~mol})$ 和 $\mathrm{POCl}_{3}(116 \mathrm{~mL})$, 升温至 $100{ }^{\circ} \mathrm{C}$ 回流 $10 \mathrm{~h}$. 停止加热. 常压蒸去剩余 $\mathrm{POCl}_{3}$, 趁热将反应液缓慢倒入 $200 \mathrm{~g}$ 碎 冰中, 搅拌, 静置. 抽滤, 固体水洗至中性, 用无水甲醇 重结晶. 抽滤, 烘干, 得 $11.3 \mathrm{~g}$ 白色粉末状固体 $7 \mathbf{a}^{[16]}$, 产率 95.8\%. m.p. $188 \sim 192{ }^{\circ} \mathrm{C} ;{ }^{1} \mathrm{H}$ NMR $(400 \mathrm{MHz}$, DMSO- $\left.d_{6}\right) \delta: 3.88(\mathrm{~s}, 3 \mathrm{H}), 3.96(\mathrm{~s}, 3 \mathrm{H}), 7.02(\mathrm{~d}, J=8.6 \mathrm{~Hz}$, 2H), $7.08(\mathrm{t}, J=8.4 \mathrm{~Hz}, 1 \mathrm{H}), 7.80 \sim 7.88(\mathrm{~m}, 2 \mathrm{H}), 8.05(\mathrm{~d}$, $J=8.6 \mathrm{~Hz}, 2 \mathrm{H}) ;{ }^{19} \mathrm{~F}$ NMR $\left(376.29 \mathrm{MHz}\right.$, DMSO- $\left.d_{6}\right) \delta:-$ 133.6; IR (KBr) v: 3063, 2949, 2844, 1623, 1495, 1469, $1416,1332,1253,1173,1133,1022,834,743,655 \mathrm{~cm}^{-1}$.

同理合成 2-(2,5-二氟-4-甲氧基苯基)-5-(4-甲氧基苯 基)-1,3,4-惡二唑(7b), 得 $11.8 \mathrm{~g}$ 白色粉末状固体 7b, 产 率 94.5\%. m.p. $183 \sim 187{ }^{\circ} \mathrm{C}$; ${ }^{1} \mathrm{H}$ NMR $(400 \mathrm{MHz}$, DMSO- $\left.d_{6}\right) \delta: 3.92(\mathrm{~s}, 3 \mathrm{H}), 4.03(\mathrm{~s}, 3 \mathrm{H}), 7.18(\mathrm{~d}, J=8.7$ $\mathrm{Hz}, 1 \mathrm{H}), 7.39(\mathrm{t}, J=8.8 \mathrm{~Hz}, 1 \mathrm{H}), 7.86 \sim 7.95(\mathrm{~m}, 2 \mathrm{H}), 8.10$ (d, $J=8.68 \mathrm{~Hz}, 2 \mathrm{H}) ;{ }^{19} \mathrm{~F}$ NMR $\left(376.29 \mathrm{MHz}\right.$, DMSO- $\left.d_{6}\right) \delta$ : $-102.4,-136.8$. IR (KBr) v: 3056, 2955, 2852, 1633, $1612,1519,1498,1470,1444,1397,1340,1260,1177$, $1159,1016,878,823,744,650,626 \mathrm{~cm}^{-1}$.

2.2.6 2-(2-氟-4-羟基苯基)-5-(4-羟基苯基)-1,3,4-恶二 唑 $(\mathbf{8 a})$ 的合成

在装有机械搅拌、带干燥管的回流冷凝管和温度计 的 $100 \mathrm{~mL}$ 三口圆底烧瓶中加入干燥的 $\mathrm{Py} \cdot \mathrm{HBr}(19.2 \mathrm{~g}$, $0.12 \mathrm{~mol})$ 和无水 $\mathrm{AlCl}_{3}(24.0 \mathrm{~g}, 0.18 \mathrm{~mol})$, 微热, 搅拌, 形成离子液体. 再加入干燥甲苯 $(30 \mathrm{~mL})$ 和干燥的化合 物 $7 \mathbf{a}(9.0 \mathrm{~g}, 0.03 \mathrm{~mol})$, 升温回流 $8 \mathrm{~h}$. 停止加热, 趁热将
反应液倒入浓盐酸 $(5 \mathrm{~mL})$ 和冰 $(100 \mathrm{~g})$ 的混合液中充分摚 拌水解, 静置. 抽滤, 无水甲醇重结晶, 抽滤, 烘干得 $7.6 \mathrm{~g}$ 白色粉末状固体 $8 \mathrm{a}^{[17]}$, 产率 93.6\%. m.p. 338 $340{ }^{\circ} \mathrm{C}$; ${ }^{1} \mathrm{H}$ NMR $\left(400 \mathrm{MHz}\right.$, DMSO- $\left.d_{6}\right) \delta: 5.37(\mathrm{~s}, 2 \mathrm{H})$, $6.63(\mathrm{~d}, J=8.4 \mathrm{~Hz}, 1 \mathrm{H}), 6.86(\mathrm{~d}, J=8.0 \mathrm{~Hz}, 2 \mathrm{H}), 7.16(\mathrm{~s}$, $1 \mathrm{H}), 7.60(\mathrm{~d}, J=8.0 \mathrm{~Hz}, 1 \mathrm{H}), 7.96(\mathrm{~d}, J=8.4 \mathrm{~Hz}, 2 \mathrm{H}) ;{ }^{19} \mathrm{~F}$ NMR $\left(376.29 \mathrm{MHz}\right.$, DMSO- $\left.d_{6}\right) \delta$ : -133.2 ; IR $(\mathrm{KBr}) v$ : $3396,3175,1610,1494,1439,835 \mathrm{~cm}^{-1}$.

同理合成 2-(2,5-二氟-4-羟基苯基)-5-(4-羟基苯 基)-1,3,4-惡二唑 $(\mathbf{8 b})$, 得 $8.1 \mathrm{~g}$ 白色粉末状固体 $8 \mathbf{b}$, 产率 92.8\%. m.p. 329 $332{ }^{\circ} \mathrm{C}$; ${ }^{1} \mathrm{H}$ NMR (400 MHz, DMSO$\left.d_{6}\right) \delta: 5.28(\mathrm{~s}, 2 \mathrm{H}), 7.15(\mathrm{~d}, J=8.7 \mathrm{~Hz}, 1 \mathrm{H}), 7.43(\mathrm{t}, J=8.5$ $\mathrm{Hz}, 1 \mathrm{H}), 7.82 \sim 7.96(\mathrm{~m}, 2 \mathrm{H}), 8.21(\mathrm{~d}, J=8.68 \mathrm{~Hz}, 2 \mathrm{H})$; ${ }^{19} \mathrm{~F}$ NMR $\left(376.29 \mathrm{MHz}\right.$, DMSO- $\left.d_{6}\right) \delta$ : $-103.8, \quad-$ 137.6; IR (KBr) v: 3161, 3063, 1609, 1588, 1495, 1438, $1321,1287,1231,1171,838,816,745,656 \mathrm{~cm}^{-1}$.

\subsection{7 目标化合物 9a $\sim 9 \mathrm{e}$ 和 $10 \mathrm{a} \sim 10 \mathrm{e}$ 合成}

在装有干燥管的回流冷凝管、100 mL 恒压滴液漏 斗、温度计和磁力搅拌器的 $100 \mathrm{~mL}$ 三口圆底烧瓶中加 入化合物 $8 \mathbf{a}(0.5 \mathrm{~g}, 1.84 \mathrm{mmol})$ 和 $40 \mathrm{~mL}$ 干燥过的吡啶, 冰浴下滴加化合物 $2 \mathrm{a}(0.71 \mathrm{~g}, 3.7 \mathrm{mmol})$. 滴毕, 升温回 流 $10 \mathrm{~h}$; 停止反应, 将反应液倒入冰-盐酸混合液中, 调 节 $\mathrm{pH}=5$, 析出大量肉色固体. 分别用 $1 \mathrm{~mol} / \mathrm{L} \mathrm{Na}_{2} \mathrm{CO}_{3}$ 溶液和水洗涤; 抽滤, 粗品经硅胶柱层析提纯 [淋洗剂: $V$ (石油醚) $: V$ (乙酸乙酯 $)=10 ： 1]$, 用石油醚重结晶, 得 $0.78 \mathrm{~g}$ 白色粉末状固体 $9 \mathrm{a}^{[18]}$, 产率 68.3\%. m.p. 150 $152{ }^{\circ} \mathrm{C} ;{ }^{1} \mathrm{H}$ NMR $\left(400 \mathrm{MHz}, \mathrm{CDCl}_{3}\right) \delta: 0.91(\mathrm{t}, J=7.8 \mathrm{~Hz}$, $6 \mathrm{H}), 1.35(\mathrm{~d}, J=8.4 \mathrm{~Hz}, 8 \mathrm{H}), 1.65 \sim 1.70(\mathrm{~m}, 4 \mathrm{H}), 2.71(\mathrm{t}$, $J=8.2 \mathrm{~Hz}, 4 \mathrm{H}), 7.23 \sim 7.29(\mathrm{~m}, 2 \mathrm{H}), 7.32(\mathrm{~d}, J=7.9 \mathrm{~Hz}$, $1 \mathrm{H}), 7.37 \sim 7.43(\mathrm{~m}, 2 \mathrm{H}), 8.10(\mathrm{~d}, J=7.1 \mathrm{~Hz}, 1 \mathrm{H}), 8.12(\mathrm{~d}$, $J=7.6 \mathrm{~Hz}, 4 \mathrm{H}), 8.16 \sim 8.20(\mathrm{~m}, 1 \mathrm{H}), 8.22(\mathrm{t}, J=7.8 \mathrm{~Hz}$, $2 \mathrm{H}) ;{ }^{19} \mathrm{~F}$ NMR $\left(376.29 \mathrm{MHz}, \mathrm{CDCl}_{3}\right) \delta:-107.10$; IR (KBr) $v$ : 3042, 2926, 2854, 1748, 1734, 1617, 1496, 1476, 1270, 1215, 1180, 1133, 1062, 764, 746, $699 \mathrm{~cm}^{-1}$; MS (35 eV) $m / z(\%): 620\left(\mathrm{M}^{+}, 9.8\right), 603$ (14.6), 579 (27.2), 447 (79.8), 175 (100). Anal. calcd for $\mathrm{C}_{38} \mathrm{H}_{37} \mathrm{FN}_{2} \mathrm{O}_{5}$ : C 73.53, H 6.01, N 4.51; found C 73.34, H 5.98, N 4.47.

同理合成 9b: 得 $0.85 \mathrm{~g}$ 白色粉末状固体, 产率 70.8\%. m.p. $112 \sim 114{ }^{\circ} \mathrm{C} ;{ }^{1} \mathrm{H}$ NMR (400 MHz, $\left.\mathrm{CDCl}_{3}\right) \delta$ : $0.89(\mathrm{t}, J=7.8 \mathrm{~Hz}, 6 \mathrm{H}), 1.30(\mathrm{t}, J=8.4 \mathrm{~Hz}, 12 \mathrm{H}), 1.62 \sim$ $1.67(\mathrm{~m}, 4 \mathrm{H}), 2.70(\mathrm{t}, J=8.0 \mathrm{~Hz}, 4 \mathrm{H}), 7.24 \sim 7.28(\mathrm{~m}, 2 \mathrm{H})$, $7.32(\mathrm{~d}, J=7.9 \mathrm{~Hz}, 1 \mathrm{H}), 7.37 \sim 7.43(\mathrm{~m}, 2 \mathrm{H}), 8.10(\mathrm{~d}, J=$ $7.1 \mathrm{~Hz}, 1 \mathrm{H}), 8.12(\mathrm{~d}, J=7.6 \mathrm{~Hz}, 4 \mathrm{H}), 8.16 \sim 8.20(\mathrm{~m}$, $1 \mathrm{H}$, ), $8.22(\mathrm{t}, J=8.2 \mathrm{~Hz}, 2 \mathrm{H}) ;{ }^{19} \mathrm{~F}$ NMR $(376.29 \mathrm{MHz}$, $\left.\mathrm{CDCl}_{3}\right) \delta:-107.35$; IR (KBr) $v: 3040,2925,2852,1745$, 
1732, 1616, 1502, 1462, 1266, 1216, 1176, 1130, 1060, 758, 744, $695 \mathrm{~cm}^{-1}$; MS (35 eV) m/z (\%): $648\left(\mathrm{M}^{+}, 10.8\right)$, 635 (14.6), 629 (100), 461 (65.7), 189 (82.1). Anal. calcd for $\mathrm{C}_{40} \mathrm{H}_{41} \mathrm{FN}_{2} \mathrm{O}_{5}$ : C 74.05, $\mathrm{H}$ 6.37, $\mathrm{N}$ 4.32; found $\mathrm{C} 73.92$, $\mathrm{H} 6.32, \mathrm{~N} 4.29$.

同理合成 9c: 得 $0.85 \mathrm{~g}$ 白色针状晶体, 产率 $69.2 \%$. m.p. $136 \sim 138{ }^{\circ} \mathrm{C} ;{ }^{1} \mathrm{H}$ NMR $\left(400 \mathrm{MHz}, \mathrm{CDCl}_{3}\right) \delta: 0.89$ (t, $J=8.2 \mathrm{~Hz}, 6 \mathrm{H}), 1.29 \sim 1.34(\mathrm{~m}, 16 \mathrm{H}), 1.67(\mathrm{t}, J=8.0 \mathrm{~Hz}$, $4 \mathrm{H}), 2.71(\mathrm{t}, J=8.4 \mathrm{~Hz}, 4 \mathrm{H}), 7.24 \sim 7.26(\mathrm{~m}, 2 \mathrm{H}), 7.34(\mathrm{t}$, $J=8.6 \mathrm{~Hz}, 4 \mathrm{H}), 7.42(\mathrm{~d}, J=8.6 \mathrm{~Hz}, 2 \mathrm{H}), 8.10 \sim 8.13(\mathrm{~m}$, $4 \mathrm{H}), 8.22 \sim 8.26(\mathrm{~m}, 3 \mathrm{H}) ;{ }^{19} \mathrm{~F}$ NMR $\left(376.29 \mathrm{MHz}, \mathrm{CDCl}_{3}\right)$ $\delta$ : - 107.16; IR (KBr) v: 3033, 2922, 2851, 1743, 1728, 1610, 1492, 1476, 1266, 1211, 1174, 1127, 1057, 758, 744, $699 \mathrm{~cm}^{-1}$; MS (35 eV) $\mathrm{m} / \mathrm{z}(\%): 676\left(\mathrm{M}^{+}, 4.8\right), 658(100)$, 633 (30), 542 (50.3), 419 (32.9). Anal. calcd for $\mathrm{C}_{42} \mathrm{H}_{45} \mathrm{~F}-$ $\mathrm{N}_{2} \mathrm{O}_{5}$ : C 74.53, H 6.70, N 4.14; found C 74.38, H 6.67, N 4.16.

同理合成 9d: 得 $0.86 \mathrm{~g}$ 白色针状晶体, 产率 $65.7 \%$. m.p. $94 \sim 95{ }^{\circ} \mathrm{C} ;{ }^{1} \mathrm{H}$ NMR $\left(400 \mathrm{MHz}, \mathrm{CDCl}_{3}\right) \delta: 0.89$ (t, $J=8.1 \mathrm{~Hz}, 6 \mathrm{H}), 1.32(\mathrm{~d}, J=7.8 \mathrm{~Hz}, 20 \mathrm{H}), 1.63 \sim 1.68(\mathrm{~m}$, $4 \mathrm{H}), 2.71(\mathrm{t}, J=8.4 \mathrm{~Hz}, 4 \mathrm{H}), 7.24 \sim 7.28(\mathrm{~m}, 2 \mathrm{H}), 7.34(\mathrm{~d}$, $J=6.8 \mathrm{~Hz}, 4 \mathrm{H}), 7.42(\mathrm{~d}, J=8.6 \mathrm{~Hz}, 2 \mathrm{H}), 8.10 \sim 8.14(\mathrm{~m}$, 4H), 8.21 8.26 (m, 3H); ${ }^{19} \mathrm{~F}$ NMR (376.29 MHz, $\left.\mathrm{CDCl}_{3}\right)$ $\delta$ : - 107.16; IR (KBr) v: 3033, 2922, 2851, 1743, 1728, 1610, 1492, 1476, 1266, 1211, 1174, 1127, 1057, 758, 744, $699 \mathrm{~cm}^{-1}$; MS (35 eV) m/z (\%): $704\left(\mathrm{M}^{+}, 5.6\right), 685$ (98.6), 661 (32), 633 (28.6), 557 (47.3). Anal. calcd for $\mathrm{C}_{44} \mathrm{H}_{49} \mathrm{FN}_{2} \mathrm{O}_{5}$ : C 74.97, H 7.01, N 3.97; found C 74.81, H 6.97, N 3.94 .

同理合成 9e: 得 $0.88 \mathrm{~g}$ 白色针状晶体, 产率 $65.1 \%$. m.p. $126 \sim 128{ }^{\circ} \mathrm{C} ;{ }^{1} \mathrm{H}$ NMR (400 MHz, $\mathrm{CDCl}_{3}$ ) $\delta: 0.88$ (t, $J=8.8 \mathrm{~Hz}, 6 \mathrm{H}), 1.30$ (t, $J=8.4 \mathrm{~Hz}, 24 \mathrm{H}), 1.66(\mathrm{t}, J=8.0$ $\mathrm{Hz}, 4 \mathrm{H}), 2.71(\mathrm{t}, J=7.6 \mathrm{~Hz}, 4 \mathrm{H}), 7.24 \sim 7.28(\mathrm{~m}, 2 \mathrm{H}), 7.34$ (d, $J=6.9 \mathrm{~Hz}, 4 \mathrm{H}), 7.42(\mathrm{~d}, J=8.6 \mathrm{~Hz}, 2 \mathrm{H}), 8.10 \sim 8.13$ (m, 4H), 8.21 8.25 (m, 3H); ${ }^{19} \mathrm{~F}$ NMR $(376.29 \mathrm{MHz}$, $\mathrm{CDCl}_{3}$ ) $\delta$ : - 107.14; IR (KBr) v: 3033, 2922, 2851, 1743, 1728, 1610, 1492, 1476, 1266, 1211, 1174, 1127, 1057, 758, 744, $699 \mathrm{~cm}^{-1}$; MS (35 eV) $\mathrm{m} / \mathrm{z}(\%): 732\left(\mathrm{M}^{+}, 4.6\right)$, 713 (97.0), 675 (40.6), 662 (18.5), 571 (47.3). Anal. calcd for $\mathrm{C}_{46} \mathrm{H}_{53} \mathrm{FN}_{2} \mathrm{O}_{5}$ : C 75.38, H 7.29, N 3.82; found C 75.23, $\mathrm{H} 7.25, \mathrm{~N} 3.85$.

同理合成 10a: 得 $0.82 \mathrm{~g}$ 白色针状晶体, 产率 69.8\%. m.p. $157 \sim 159{ }^{\circ} \mathrm{C} ;{ }^{1} \mathrm{H}$ NMR (400 MHz, $\left.\mathrm{CDCl}_{3}\right) \delta$ : $0.91(\mathrm{t}, J=8.2 \mathrm{~Hz}, 6 \mathrm{H}), 1.34 \sim 1.39(\mathrm{~m}, 8 \mathrm{H}), 1.63 \sim 1.71$ (m, 4H), $2.71(\mathrm{t}, J=8.4 \mathrm{~Hz}, 4 \mathrm{H}), 7.32 \sim 7.36(\mathrm{~m}, 4 \mathrm{H}), 7.42$ (d, $J=8.6 \mathrm{~Hz}, 2 \mathrm{H}), 8.01 \sim 8.05(\mathrm{~m}, 2 \mathrm{H}), 8.11 \sim 8.13(\mathrm{~m}$, 4H), $8.23(\mathrm{~d}, J=8.7 \mathrm{~Hz}, 2 \mathrm{H}) ;{ }^{19} \mathrm{~F}$ NMR $(376.29 \mathrm{MHz}$, $\mathrm{CDCl}_{3}$ ) $\delta:-112.53,-130.73$; IR (KBr) v: 3036, 2954, 2927, 2857, 1742, 1609, 1485, 1285, 1204, 1179, 1145, 1068, 1016, 817, $745 \mathrm{~cm}^{-1}$; MS (35 eV) $\mathrm{m} / z(\%): 638\left(\mathrm{M}^{+}\right.$, 22.3), 622 (10.7), 612 (18.2), 526 (58.0). Anal. calcd for $\mathrm{C}_{38} \mathrm{H}_{36} \mathrm{~F}_{2} \mathrm{~N}_{2} \mathrm{O}_{5}$ : C 71.46, H 5.68, N 4.39; found C 71.27, H 5.63, N 4.36.

同理合成 $10 \mathrm{~b}$ : 得 $0.81 \mathrm{~g}$ 白色针状晶体, 产率 65.8\%. m.p. $142 \sim 143{ }^{\circ} \mathrm{C} ;{ }^{1} \mathrm{H} \mathrm{NMR}\left(400 \mathrm{MHz}, \mathrm{CDCl}_{3}\right) \delta$ : $0.90(\mathrm{t}, J=7.9 \mathrm{~Hz}, 6 \mathrm{H}), 1.33 \sim 1.38(\mathrm{~m}, 12 \mathrm{H}), 1.62 \sim 1.70$ (m, 4H), $2.71(\mathrm{t}, J=8.2 \mathrm{~Hz}, 4 \mathrm{H}), 7.30 \sim 7.35(\mathrm{~m}, 4 \mathrm{H}), 7.42$ (d, $J=8.6 \mathrm{~Hz}, 2 \mathrm{H}), 8.00 \sim 8.04(\mathrm{~m}, 2 \mathrm{H}), 8.11 \sim 8.15(\mathrm{~m}$, $4 \mathrm{H}), 8.23(\mathrm{~d}, J=8.7 \mathrm{~Hz}, 2 \mathrm{H}) ;{ }^{19} \mathrm{~F}$ NMR $(376.29 \mathrm{MHz}$, $\left.\mathrm{CDCl}_{3}\right) \delta:-112.55,-130.76$; IR (KBr) v: 3036, 2954, 2927, 2857, 1742, 1609, 1485, 1285, 1204, 1179, 1145, 1068, 1016, 817, $745 \mathrm{~cm}^{-1}$; MS (35 eV) $\mathrm{m} / z(\%): 668$ (4.5), 649 (17.8), 636 (4.60), 609 (100), 554 (48.3). Anal. calcd for $\mathrm{C}_{40} \mathrm{H}_{40} \mathrm{~F}_{2} \mathrm{~N}_{2} \mathrm{O}_{5}$ : C 72.05, H 6.05, N 4.20; found C 71.83, H 6.08, N 4.15.

同理合成 10c: 得 $0.83 \mathrm{~g}$ 白色针状晶体, 产率 $64.5 \%$. m.p. $120 \sim 122{ }^{\circ} \mathrm{C} ;{ }^{1} \mathrm{H}$ NMR $\left(400 \mathrm{MHz}, \mathrm{CDCl}_{3}\right) \delta: 0.90(\mathrm{t}$, $J=8.4 \mathrm{~Hz}, 6 \mathrm{H}), 1.32 \sim 1.37(\mathrm{~m}, 16 \mathrm{H}), 1.60 \sim 1.1 .69(\mathrm{~m}$, $4 \mathrm{H}), 2.71(\mathrm{t}, J=8.6 \mathrm{~Hz}, 4 \mathrm{H}), 7.32 \sim 7.36(\mathrm{~m}, 4 \mathrm{H}), 7.42(\mathrm{~d}$, $J=8.6 \mathrm{~Hz}, 2 \mathrm{H}), 7.96 \sim 8.03(\mathrm{~m}, 2 \mathrm{H}), 8.10 \sim 8.13(\mathrm{~m}, 4 \mathrm{H})$, $8.23(\mathrm{~d}, J=8.7 \mathrm{~Hz}, 2 \mathrm{H}) ;{ }^{19} \mathrm{~F}$ NMR $\left(376.29 \mathrm{MHz}, \mathrm{CDCl}_{3}\right)$ $\delta$ : $-112.48,-130.70$; IR (KBr) v: 3036, 2954, 2927, 2857, 1742, 1609, 1485, 1285, 1204, 1179, 1145, 1068, 1016, 817, $745 \mathrm{~cm}^{-1}$; MS (35 eV) m/z (\%): $694\left(\mathrm{M}^{+}, 100\right)$, 676 (41), 665 (4.8), 638 (4.6), 526 (4.6). Anal. calcd for $\mathrm{C}_{42} \mathrm{H}_{44} \mathrm{~F}_{2} \mathrm{~N}_{2} \mathrm{O}_{5}$ : C 72.60, H 6.38, N 4.03; found C 72.41, H $6.42, \mathrm{~N} 4.06$.

同理合成 $10 \mathrm{~d}$ : 得 $0.84 \mathrm{~g}$ 白色针状晶体, 产率 62.7\%. m.p. $138 \sim 140{ }^{\circ} \mathrm{C} ;{ }^{1} \mathrm{H} \mathrm{NMR}\left(400 \mathrm{MHz}, \mathrm{CDCl}_{3}\right) \delta$ : $0.91(\mathrm{t}, J=7.6 \mathrm{~Hz}, 6 \mathrm{H}), 1.32 \sim 1.38(\mathrm{~m}, 20 \mathrm{H}), 1.60 \sim 1.68$ (M, 4H), $2.72(\mathrm{t}, J=8.6 \mathrm{~Hz}, 4 \mathrm{H}), 7.31 \sim 7.36(\mathrm{~m}, 4 \mathrm{H}), 7.42$ $(\mathrm{d}, J=8.6 \mathrm{~Hz}, 2 \mathrm{H}), 7.99 \sim 8.04(\mathrm{~m}, 2 \mathrm{H}), 8.10 \sim 8.13(\mathrm{~m}$, $4 \mathrm{H}), 8.23(\mathrm{~d}, J=8.7 \mathrm{~Hz}, 2 \mathrm{H}) ;{ }^{19} \mathrm{~F}$ NMR $(376.29 \mathrm{MHz}$, $\left.\mathrm{CDCl}_{3}\right) \delta$ : $-112.50,-130.74$; IR (KBr) v: 3036, 2954, 2927, 2857, 1742, 1609, 1485, 1285, 1204, 1179, 1145, 1068, 1016, 817, $745 \mathrm{~cm}^{-1}$; MS (35 eV) $\mathrm{m} / \mathrm{z}(\%): 722\left(\mathrm{M}^{+}\right.$, 10.9), 704 (58.2), 693 (6.7), 666 (7.2), 610 (38.5\%). Anal. calcd for $\mathrm{C}_{44} \mathrm{H}_{48} \mathrm{~F}_{2} \mathrm{~N}_{2} \mathrm{O}_{5}$ : C 73.11, H 6.69, N 3.88; found C 73.02, H 6.57, N 3.92 .

同理合成 10e: 得 $0.88 \mathrm{~g}$ 白色针状晶体, 产率 $63.6 \%$. 
m.p. $144 \sim 145{ }^{\circ} \mathrm{C} ;{ }^{1} \mathrm{H}$ NMR $\left(400 \mathrm{MHz}, \mathrm{CDCl}_{3}\right) \delta: 0.91$ (t, $J=8.2 \mathrm{~Hz}, 6 \mathrm{H}), 1.33 \sim 1.38(\mathrm{~m}, 24 \mathrm{H}), 1.60 \sim 1.1 .69(\mathrm{~m}$, $4 \mathrm{H}), 2.72(\mathrm{t}, J=8.4 \mathrm{~Hz}, 4 \mathrm{H}), 7.31 \sim 7.36(\mathrm{~m}, 4 \mathrm{H}), 7.42(\mathrm{~d}$, $J=8.6 \mathrm{~Hz}, 2 \mathrm{H}), 8.01 \sim 8.05(\mathrm{~m}, 2 \mathrm{H}), 8.11 \sim 8.13(\mathrm{~m}, 4 \mathrm{H})$, $8.23(\mathrm{~d}, J=8.7 \mathrm{~Hz}, 2 \mathrm{H}) ;{ }^{19} \mathrm{~F}$ NMR $\left(376.29 \mathrm{MHz}, \mathrm{CDCl}_{3}\right)$ $\delta$ : $-112.55,-130.78$; IR (KBr) v: 3036, 2954, 2927, 2857, 1742, 1609, 1485, 1285, 1204, 1179, 1145, 1068, 1016, 817, $745 \mathrm{~cm}^{-1}$; MS (35 eV) m/z (\%): 751 (26.8), 732 (82.5), 722 (6.7), 695 (5.6), 636 (38.5), 581 (8.5). Anal. calcd for $\mathrm{C}_{46} \mathrm{H}_{52} \mathrm{~F}_{2} \mathrm{~N}_{2} \mathrm{O}_{5}$ : C 73.58, H 6.98, N 3.73; found C 73.46, H 6.92, N 3.76 .

辅助材料(Supporting Information) 目标化合物的 IR、 ${ }^{1} \mathrm{H} N M R 、{ }^{19} \mathrm{~F} N M R 、 M S$ 谱图, 以及 DSC 曲线. 这些材 料可以免费从本刊网站(http://sioc-journal.cn/)上下载.

\section{References}

[1] Nori, T.; Sekine, T.; Watanabe, J. J. Mater. Chem. 1996, 6, 1231

[2] Link, D. R.; Natale, G.; Shao, R. F. Science 1997, 278, 1924.

[3] Madsen, L. A.; Dingemans, T. J.; Nakata, M. Phys. Rev. Lett. 2004, 92, 145506.

[4] Taushanoff, S.; Le, K. V.; Williams, J. J. Mater. Chem. 2010, 20, 5893.

[5] Prasad, V.; Kang, S. W.; Suresh, K. A. J. Am. Chem. Soc. 2005, 127, 17224 .
[6] Gleeson, H. F.; Kaur, S.; Görtz, V. ChemPhysChem 2014, 15 , 1251.

[7] Du, Q.; You, H.-J.; Wang, X.-Y.; Zhang, Z.-Y.; Dai, Z.-Q. Liq. Cryst. Disp. 2011, 26, 719 (in Chinese). (杜琼, 游红军, 汪晓燕, 张智勇, 戴志群，液晶与显示, 2011, 26, 719.)

[8] Görtz, V.; Southern, C.; Roberts, N. W.; Gleeson, H. F.; Goodby, J. W. Soft Matter 2009, 5, 463.

[9] Cooper, L. L.; Samulski, E. T.; Scharrer, E. Mol. Cryst. Liq. Cryst. 2009, 511, 203.

[10] Speetjens, F.; Lindborg, J.; Tauscher, T. J. Mater. Chem. 2012, 22, 22558.

[11] Wang, Y.-L.; Du, Q.; Wang, G.-H.; Zhang, Z.-Y. Chin. J. Org. Chem. 2013, 33, 2010 (in Chinese).

(王勇丽，杜琼，王国华，张智勇，有机化学，2013，33, 2010.)

[12] Maksimenko, S. I.; Novikove, N. S.; Yarkove, M. Y. Russ. J. Org. Chem. 2007, 43, 1773.

[13] Yoshida, M.; Otaka, H.; Doi, T. Eur. J. Org. Chem. 2014, 27, 6010.

[14] Huang, H.-M.; Yu, H,-T.; Chen, P.-L. Chin. J. Org. Chem. 2004, 24, 502 (in Chinese). (黄华鸣, 于海涛, 陈培丽, 有机化学, 2004, 24, 502.)

[15] Neubert, M. E. Mol. Cryst. Liq. Cryst. 1979, 53, 101.

[16] Zhu, D.-X.; Lu, H.-F.; Zhang, J. CN 101643456, 2009 [Chem. Abstr. 2009, 152, 287388].

[17] You, H.-J. Bachelor Thesis, Wuhan Polytechnic University, Wuhan, 2009 (in Chinese). (游红军, 学士论文, 武汉工业学院, 武汉, 2009.)

[18] Huang, P.; Hu, P.-L.; Shen, D. Liq. Cryst. Disp. 2010, 25, 626 (in Chinese)

(黄佩, 胡萍兰, 沈冬, 液晶与显示, 2010, 25, 626.)

(Li, L.; Lu, Z.) 